\title{
SOBREVIDA EN CARCINOMA ESPINOCELULAR DE MUCOSA ORAL: ANÁLISIS DE 161 PACIENTES*
}

\author{
Drs. Bárbara Momares D. ${ }^{1}$, Gabriela Contreras C. ${ }^{1}$, Benjamín Martínez R. ${ }^{1}$, \\ Nicolás Ávalos J. ${ }^{2}$, Leonardo Carmona R. ${ }^{3}$ \\ 1 Departamento de Patología Oral. Universidad Mayor. \\ 2 Departamento de Cirugía en Cabeza y Cuello. Hospital Fuerza Aérea de Chile, Universidad Mayor. \\ 3 Departamento de Radioterapia. Fundación Arturo López Pérez. \\ Santiago, Chile.
}

\begin{abstract}
Survival in oral mucosa squamous cell carcinoma. Analysis of 161 patients

Introduction: Oral Cancer accounts 3\% of all human cancers. Squamous Cell Carcinoma is the most common. Nowadays, in Chile and the world, oral cancer is reaching relevance because of its high incidence and low survival rates; most often is squamous cell carcinoma (SCC). Aim: We analyzed the five-year survival of 161 patients diagnosed with SCC. Also was studied relationships between survival and gender, age, location, tobacco, alcohol and treatment. Methods: Retrospective study among 1989 and 2010. The study population was mainly men gender (59\%), average age 64 years and location of the CEC preferably in tongue (37.6\%). 54\% were well differentiated carcinomas and TNM, the majority corresponding to T2N0M0. Kaplan-Meier curve and Log Rank test were used for survival analysis and to compare them. We determined significant difference when $p$ value $<0.05$. Results: The overall survival at five years was $46 \%$, the lowest rates were in the male gender, age group 60, floor of mouth, smokers and alcohol consumers. Conclusions: Although tobacco and alcohol are factors highly related to the presence of CEC, there was no significant difference between survival and these habits. There were differences between the survival curves for patients treated with surgery associated with radiotherapy in comparison to other treatments.
\end{abstract}

Key words: Survival, squamous cell carcinoma, oral cancer.

\section{Resumen}

Introducción: Hoy en día, en Chile y el mundo, el cáncer oral ha tomado mayor importancia por su incidencia y bajos porcentajes de sobrevida, el más frecuente corresponde al Carcinoma Espinocelular (CEC). Objetivo: Analizar la sobrevida a cinco años de 161 pacientes de la Facultad de Odontología de la Universidad Mayor y Fundación Arturo López Pérez (FALP), diagnosticados con CEC de mucosa oral mediante

*Recibido el 4 de abril de 2014 y aceptado para publicación el 7 de mayo de 2014.

Se obtuvo autorización del comité de ética de la FALP para realizar este estudio.

Los autores no refieren conflictos de interés.

Correspondencia: Dr. Nicolás Ávalos J. contacto@cabezaycuello.cl 
biopsia. Se analizó cómo afecta a la sobrevida, el género, edad, localización, consumo de tabaco y/o alcohol y tratamiento. Material y Método: Estudio retrospectivo entre los años 1989 y 2010. La población estudiada correspondía principalmente a hombres (59\%), edad promedio de 64 años y ubicación del CEC preferentemente en lengua (37,6\%). El 54\% eran carcinomas bien diferenciados y, según TNM, la mayor parte correspondía a T2N0M0. Se utilizó para los análisis de sobrevida la curva de Kaplan-Meier y Log Rank Test para compararlas. Se determinó si existían diferencias significativas cuando el valor de $\mathrm{p}<0,05$. Resultados: $\mathrm{La}$ sobrevida general a los cinco años fue de $46 \%$, las tasas más bajas fueron en el género masculino, el grupo mayores de 60 años, en piso de boca, fumadores y bebedores de alcohol. Conclusiones: A pesar que el tabaco y el alcohol son factores altamente relacionados a la presencia de CEC, no se observó diferencia significativa entre la sobrevida y estos hábitos. Hubo diferencia entre las curvas de sobrevida de pacientes tratados con cirugía asociada a radioterapia en relación a otros tratamientos.

Palabras clave: Sobrevida, carcinoma espinocelular, cáncer oral.

\section{Introducción}

El cáncer oral representa el 3\% de todos los cánceres que nos afectan. Cada año se diagnostican en el mundo más de 575.000 casos nuevos, en Chile se estima que la morbilidad por cáncer oral y faríngeo corresponde aproximadamente al $1,6 \%$ del total de todos los cánceres ${ }^{1,2}$, cifra algo menor a la de Estados Unidos ${ }^{3}$.

La cavidad oral se define como el territorio entre la cara mucosa de los labios y el istmo de las fauces (pilar anterior) que la separa de la orofaringe. Los subsitios que contiene la cavidad oral son: labio, 2/3 anteriores de la lengua, piso de la boca, gíngiva, cara interna de la mejilla, trígono retromolar y paladar duro. La cavidad oral está revestida por epitelio pluriestratificado y contiene glándulas salivares menores. Dentro del cáncer oral, el carcinoma espinocelular es el más frecuente $(90 \%)^{4,5}$. Corresponde a una neoplasia de etiología multifactorial, entre sus factores predisponentes se encuentra el tabaco, alcohol e infección por virus papiloma, los que incluso pueden tener efecto acumulativo en el tiempo ${ }^{1,6-8}$.

La edad también se considera factor de riesgo, $90 \%$ son diagnosticados en pacientes mayores de 45 años, sin embargo, ha aumentado el número de adultos entre 20-30 años que han desarrollado cáncer, especialmente en lengua ${ }^{9}$. Incluso tienden a presentar mayor tasa loco-regional de recurrencia y tasa de sobrevida menor ${ }^{4}$.

La mayoría de los casos se presentan en hombres, pero el aumento del consumo de tabaco en mujeres ha producido mayor incidencia en ellas, alcanzando la relación $\mathrm{H}: \mathrm{M} 2: 1^{10,11}$.

La manifestación clínica es variada, en estadíos tempranos se puede observar una lesión leucoplásica, eritroplásica, leucoeritroplásica o una úlcera pequeña asintomática. En etapas más avanzadas puede presentarse como masa exofítica o endofítica, con borde irregular, indurada y de fondo sucio ${ }^{12,13}$.

El diagnóstico temprano es difícil debido a la poca sintomatología que presenta, esto provoca que la mayoría de los pacientes consulten cuando las lesiones han alcanzado gran tamaño, siendo entonces el tratamiento más complicado y el pronóstico peor $^{14}$.

El tratamiento de un paciente oncológico es multidisciplinario ${ }^{15}$. Existen diferentes modalidades de tratamiento; cirugía, radioterapia y quimioterapia, que pueden ser aplicadas en conjunto o por sí solas $^{16,10,17}$.

A pesar de los avances en las técnicas quirúrgicas y terapias ayudantes los porcentajes de sobrevida se han mantenido relativamente estables. En Estados Unidos, según Surveillance Epidemiology and End Results (SEER) Cancer Statistics Review, entre 1957-2008 la sobrevida a los cinco años para ambos sexos fue $61,5 \%{ }^{18}$.

El propósito de este trabajo es analizar el porcentaje de sobrevida a los cinco años de una muestra de pacientes diagnosticados con Carcinoma Espinoceluarar de mucosa oral (CEC) y observar su relación con ciertas características epidemiológicas.

\section{Material y Método}

Se revisa la base de datos del departamento de Patología Oral, (Escuela de Odontología Universidad Mayor) y de pacientes tratados en radioterapia de la Fundación Arturo López Pérez (FALP). Criterios de inclusión; pacientes diagnosticados con Carcinoma Espinocelular (CEC) de mucosa oral mediante biopsia. Se excluyen otras localizaciones y otros tipos histopatológicos. Se obtiene autorización del comité de ética FALP para realizar estudio.

La muestra corresponde a pacientes diagnosticados entre diciembre de 1989 y diciembre de 2010, se analizan características epidemiológicas y se determina la sobrevida a los cinco años según causa de muerte por CEC o por otras causas.

Se recolectan datos: nombre, edad (años), teléfono, género ( $\mathrm{M}$ o F), alcohol (sí, no), tabaco (sí, no), grado de diferenciación (bien, moderado y poco 
diferenciado), localización (labio, lengua, paladar duro, piso de boca, encía, otras), clasificación TNM, si se realizó o no tratamiento, tipo de éste (cirugía y quimioterapia/cirugía y radioterapia/cirugía, radioterapia y quimioterapia, otros). Los datos se manejaron de forma anónima y reservada.

Se determinó los pacientes fallecidos mediante certificado de defunción con causa de muerte, obtenido del Registro Civil de Chile. Los pacientes que permanecieron vivos fueron estudiados a partir del 14 de septiembre del año 2011 retrospectivamente hasta su fecha de diagnóstico.

Se analizó relación existente entre tiempo de sobrevida y presencia de factores más relevantes. Se utilizó para los análisis de sobrevida la curva de Kaplan Meier. Se utilizó test de rango de logaritmo para comparar las curvas de sobrevida. Se determinó si existían diferencias significativas cuando el valor de $\mathrm{p}<0,05$.

\section{Resultados}

\section{Características epidemiológicas}

Se obtuvo muestra de 217 pacientes, 128 (59\%) hombres y 89 (41\%) mujeres, entregando relación hombre: mujer 1,4: 1. La edad de los pacientes iba desde 15 a 96 años, promedio de $64 \pm 14,4$ años (x $\pm \mathrm{ds})$. Se agruparon las edades en tres rangos; 21 $(9,8 \%)$ eran menores de 45 años, 49 (22,9\%) entre 45 y 60 años y $144(67,3 \%)$ mayores de 60 años (Tabla 1).

En 146 pacientes se pudo evaluar el hábito de fumar, de ellos $100(68,5 \%)$ fumaban. En el caso de los pacientes fumadores, 73 eran hombres $(73 \%)$ y 27 eran mujeres (27\%). No hubo información con respecto a virus papiloma (VPH). No hubo claridad de registro para identificar segundos primarios y tumores sincrónicos.

El hábito de beber alcohol se estudió en 139 pacientes. De ellos $56(40,3 \%)$ no bebía alcohol y $83(59,7 \%)$ sí lo hacía, principalmente hombres $(80,7 \%)$.

En relación a ubicaciones registradas en 210 $(96,7 \%)$ pacientes, los sitios más frecuentes fueron; lengua en 79 casos $(37,6 \%)$, encía y reborde; 36 casos $(17,1 \%)$ seguidos por piso de boca; 35 casos $(16,7 \%)$ (Tabla 1).

Respecto al grado de diferenciación analizados en 215 biopsias, 116 (54\%) eran bien diferenciados, 79 $(36,7 \%)$ moderadamente diferenciados y $20(9,3 \%)$ poco diferenciados.

De acuerdo a la clasificación TNM obtenida en 84 pacientes, se observó que $12(14,3 \%)$ presentaron T1, $40(47,6 \%)$ T2, 13 (15,5\%) T3 у $19(22,6 \%)$ T4. $\mathrm{Al}$ estudiar $(\mathrm{N})$ se observó 46 (54,8\%) N0, 21 (25\%)
Tabla 1. Características demográficas de los pacientes con CEC

\begin{tabular}{|lrr|}
\hline Variable & n & \% \\
Edad $(\mathrm{n}=214)$ & & \\
$<45$ & 21 & 9,8 \\
$45-60$ & 49 & 22,9 \\
$>60$ & 144 & 67,3 \\
Sexo $(\mathrm{n}=217)$ & & \\
Femenino & 89 & 41,0 \\
Masculino & 128 & 59,0 \\
Hábitos (n=145) & & \\
Tabaco & 27 & 18,6 \\
Alcohol & 10 & 6,9 \\
Tabaco y alcohol & 73 & 50,3 \\
Sin hábitos & 35 & 24,1 \\
Localización (n=210) & & \\
Labio & 15 & 7,1 \\
Lengua & 79 & 37,6 \\
Piso de boca & 35 & 16,7 \\
Encía y reborde & 36 & 17,1 \\
Mejilla & 12 & 5,7 \\
Paladar duro-blando & 18 & 8,6 \\
Lengua y piso de boca & 11 & 5,2 \\
Otros & 4 & 1,9 \\
\hline
\end{tabular}

Tabla 2. Distribución según tratamientos $(n=138)$

\begin{tabular}{|lcc|}
\hline Tratamiento & $\mathbf{n}$ & $\mathbf{\%}$ \\
Cirugía-RT-QMT & 29 & 21,0 \\
Cirugía-radioterapia & 79 & 57,2 \\
Otros & 29 & 21,0 \\
\hline
\end{tabular}

con N1, $15(17,9 \%)$ con N2 y $2(2,4 \%)$ con N3. Al analizar (M) se observó que de 84 pacientes sólo dos $(2,4 \%)$ presentaron metástasis.

\section{Tratamiento}

En 143 pacientes se evaluó si recibieron tratamiento o no, $96,5 \%$ se realizó tratamiento. El más frecuente fue asociación de cirugía con radioterapia aplicada en 79 pacientes $(57,2 \%), 29$ casos $(21 \%)$ recibieron cirugía-quimioterapia-radioterapia y 29 pacientes $(21 \%)$ recibieron otros tratamientos, principalmente braquiterapia y tratamientos paliativos del dolor (Tabla 2).

\section{Sobrevida}

Del total de la muestra, se pudo evaluar sobrevida en 161 pacientes. Logrando un seguimiento de hasta 6.966 días, mediana de 289 días y mínimo de 35 días. 
Al relacionar pacientes fallecidos con género, $65,3 \%$ eran hombres y $34,7 \%$ mujeres. No hubo diferencia significativa entre ambos sexos $\left(\chi^{2}=3,058\right.$; $\mathrm{p}=0,08)$.

Al realizar análisis de Kaplan-Meier, la sobrevida a los 5 años fue $46 \%$, a los 10 años fue $34,3 \%$ y a los 14 años fue 15,1\% (Figura 1). El tiempo medio de sobrevida fue 6,9 años. Se logró estimar que el $25 \%$ de la muestra se mantuvo viva a los 12 años, el 50\% a los 4,6 años y el $75 \%$ al año.

En sobrevida y edad (evaluada en 160 pacientes) se observó que el grupo de menores de 45 años tuvo mayor tiempo promedio de sobrevida: 6,8 años. El grupo entre 45-60 años presentó promedio de sobrevida de 6,4 años y sobrevida a los cinco años de $50,2 \%$. Por último, el grupo de mayores de 60 años, arrojó promedio de sobrevida de 6,7 años y sobrevida a los cinco años de $44,1 \%$ (Figura 2). No se observó diferencia significativa entre los distintos grupos etarios y la sobrevida (Log Rank test, $\chi^{2}=0,36 ; p=0,837$ ).

Al relacionar sobrevida y género en 161 pacientes, se vio que a los cinco años la sobrevida era $56,6 \%$ en mujeres y $38,3 \%$ en hombres (Figura 3 ). El tiempo de sobrevida promedio para mujeres fue 8,3 años y en hombres 6,1 años. No se observó diferencia significativa en relación al género y sobrevida (Log Rank test, $\chi^{2}=3,17 ; p=0,0749$ ).
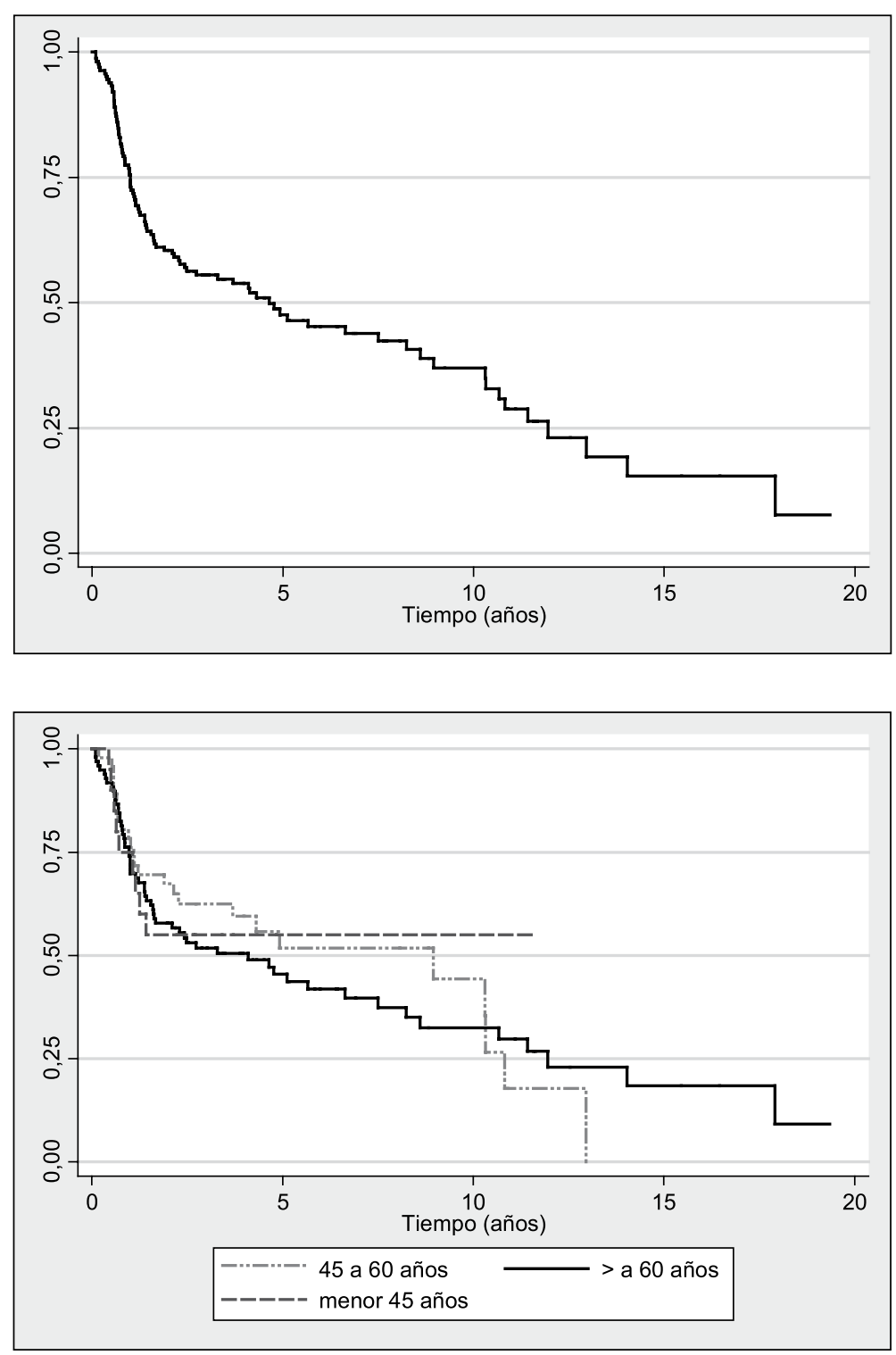

Figura 1. Curva de sobrevida KaplanMeier.
Figura 2. Curva sobrevida KaplanMeier según grupos etarios (menor 45 años, $\mathrm{n}=20 ; 45$ a 60 años, $\mathrm{n}=44$; $\mathrm{y}$, $>$ a 60 años, $n=96$ ). 
La relación entre sobrevida y localización anatómica en 155 pacientes, mostró que la mayor sobrevida fue labio con promedio de 11,3 años, lengua con 7,3 años, encía y reborde 6 años, paladar duroblando 5,8 años, asociación de lengua y piso de boca 5 años, mejilla 4,6 años y por último piso de boca con promedio 4,2 años. No se observó diferencia significativa en relación a sobrevida y localización anatómica (Log Rank test, $\chi^{2}=6,87 ; \mathrm{p}=0,443$ ). No se hizo comparación entre las distintas ubicaciones anatómicas dado que algunas correspondían a pocos casos.

$\mathrm{Al}$ analizar consumo de tabaco con sobrevida en 135 pacientes, los no fumadores tuvieron promedio de sobrevida de 8,8 años y, a los 14 años, la mitad de ellos se encontraba vivo. Respecto a fumadores se determinó tiempo promedio de sobrevida de 5,8 años y a los cinco años sobrevida de $44,4 \%$ (Figura 4). No hay diferencia significativa (Log Rank test, $\left.\chi^{2}=0,79 ; p=0,373\right)$.

El consumo de alcohol en relación con sobrevida fue analizado en 132 pacientes. Los no bebedores alcanzaron 8,1 años de sobrevida promedio y $50 \%$ de la muestra se mantuvo viva a los 14 años desde el diagnóstico. Con respecto a los bebedores se observó que la sobrevida a los cinco años fue 43,1\%, siendo el promedio de sobrevida seis años (Figura 5). No hubo diferencia significativa en relación
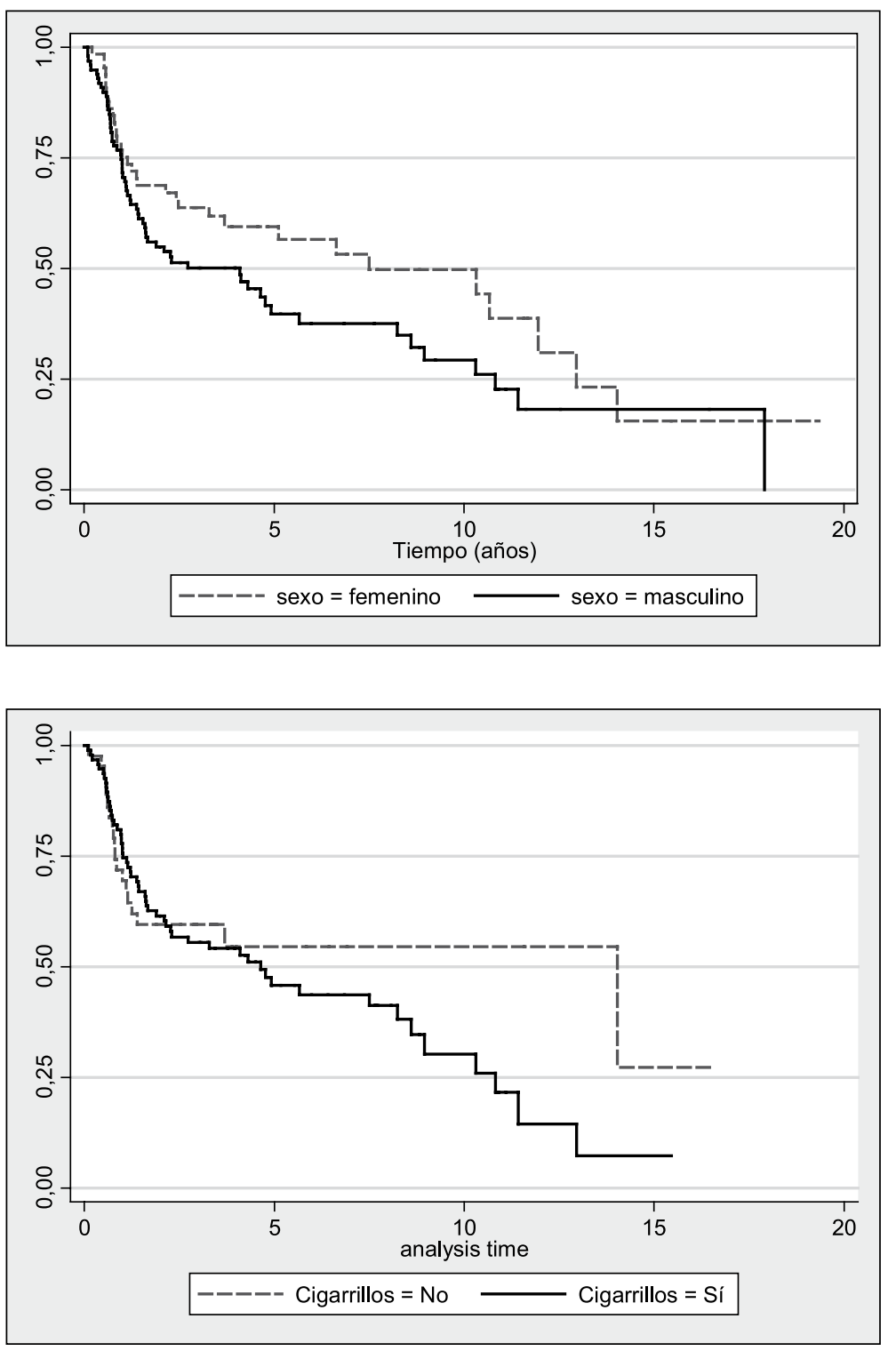

Figura 3. Curva de sobrevida KaplanMeier según género (Femenino, $\mathrm{n}=65$; Masculino, $\mathrm{n}=96$ ).
Figura 4. Curva de sobrevida KaplanMeir en relación al consumo de tabaco (Fumadores, $\mathrm{n}=92$; No fumadores, $n=43)$. 


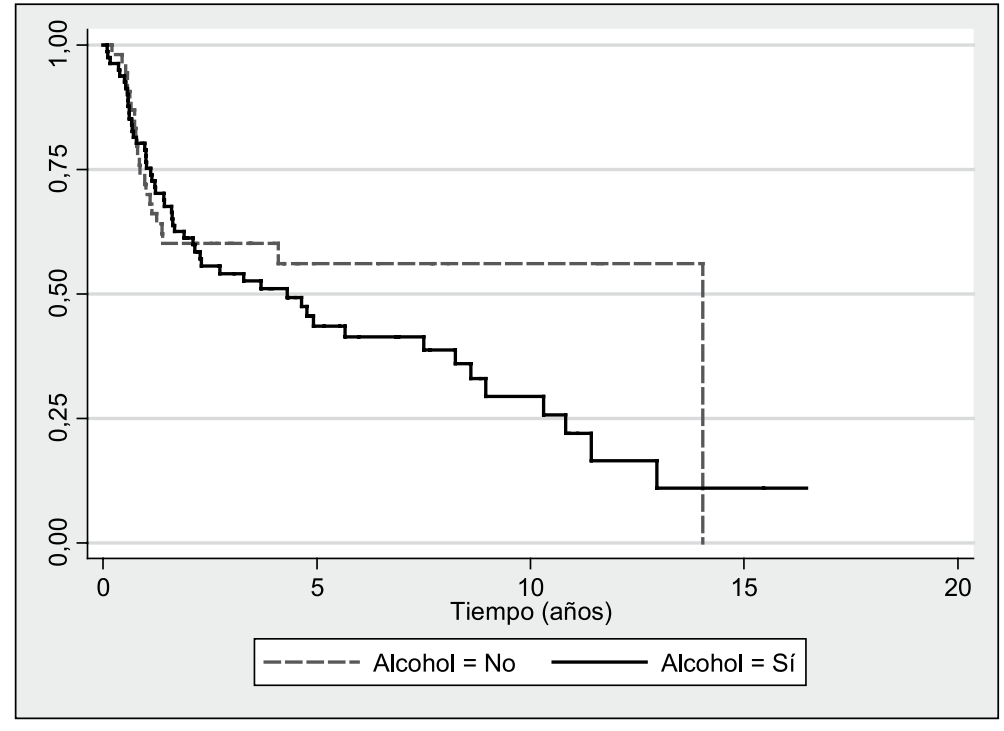

Figura 5. Curva de sobrevida KaplanMeier en relación al consumo de alcohol (Bebedores, $\mathrm{n}=80$; No bebedores, $\mathrm{n}=52)$. al consumo de alcohol y sobrevida (Log Rank test, $\left.\chi^{2}=1,09 ; \mathrm{p}=0,296\right)$.

Respecto al tratamiento y sobrevida estudiada en 138 pacientes, se determinó que los pacientes tratados con cirugía y radioterapia presentaron sobrevida a los cinco años de $47,8 \%$ y sobrevida promedio de 6,8 años. Los pacientes que recibieron otro tipo de tratamiento alcanzaron $40,3 \%$ de sobrevida a los cinco años y promedio de sobrevida 5,8 años. Se observó que existía diferencia significativa entre ambas curvas (Log Rank test, $\chi^{2}=3,849 ; p=0,05$ ).

Los pacientes que se lograron seguir hasta el final del estudio fueron 170 , de ellos $38,8 \%$ se mantuvieron vivos y $61,2 \%$ fallecieron. De estos últimos, un $56,5 \%$ la causa de muerte fue carcinoma espinocelular y $4,7 \%$ fue por otras causas (Tabla 3 ).

\section{Discusión}

En nuestro trabajo tuvimos serias limitantes debido a la falta de registro adecuado en las fichas clínicas y base de datos de los paciente; perdiendo mucha información relevante para un análisis más detallado. Aún así destacamos la importancia de tener un análisis de sobrevida chilena para una localización anatómica (cavidad oral) y un tipo histológico (carcinoma espinocelular).

El perfil epidemiológico indicó que los hombres son más afectados que las mujeres, tal como se menciona en otros estudios ${ }^{19,20}$. La relación H:M fue $1,4: 1$, similar a lo observado por Iamaroon y cols ${ }^{21}$. El $67,3 \%$ de los pacientes diagnosticados eran mayores de 60 años, media de 64,4 años, similar a otros
Tabla 3. Distribución de pacientes fallecidos y vivos $(\mathbf{n}=170)$

\begin{tabular}{|lrr|}
\hline Variable & n & \% \\
Fallecidos por CEC & 96 & 56,5 \\
Fallecidos por otra causa & 8 & 4,7 \\
Vivos & 66 & 38,8 \\
\hline
\end{tabular}

estudios $^{22,23}$. El 9,8\% de la muestra eran menores de 45 años, cercano al $12,8 \%$ que reportó estudio del año $2008^{24}$.

La histopatología más frecuente coincide con el reporte de otros autores ${ }^{25-27}$, la mayoría eran tumores bien diferenciados al momento del diagnóstico. En cambio, Guzmán y cols ${ }^{23}$, encontró que más de la mitad eran moderadamente diferenciados.

Distintos autores ${ }^{28-31}$, mencionan la lengua como ubicación más común, esto concuerda con nuestro análisis. Otros estudios ${ }^{24,19}$, han concluido que labio sería el sitio más frecuente. Pero hay que analizar con cuidado la información, ya que la cara no mucosa del labio se comporta como cáncer de piel (alta incidencia) y sólo la porción mucosa del labio se comporta como cáncer de cavidad oral.

El consumo de tabaco y alcohol son reconocidos como factores de riesgo del CEC, en este estudio comprobamos que un alto porcentaje de pacientes presentaban ambos hábitos (49,3\%).

El consumo de alcohol se presentó en $59,7 \%$ de los pacientes, similar al 63,3\% que demostró Meza y cols ${ }^{11}$. Otro estudio ${ }^{22}$, lo encontró asociado sólo en un $29,8 \%$. 
Un alto porcentaje de pacientes evaluados $(96,5 \%)$ recibieron tratamiento, siendo el más frecuente la cirugía asociada a radioterapia $(57,2 \%)$, similar a otro estudio ${ }^{32}$ en Chile, donde $62,04 \%$ recibieron la misma terapia combinada. A diferencia de esto, Peña y cols ${ }^{31}$, reveló que sólo $15,2 \%$ recibió cirugía y radioterapia. Un estudio realizado en 587 pacientes de Tailandia ${ }^{21}$, mostró radioterapia exclusiva (46\%) como tratamiento más común y sólo $33 \%$ cirugía con radioterapia.

Es importante mencionar un posible sesgo con respecto al resultado de la terapia en este estudio, ya que el $55,1 \%$ de quienes recibieron tratamiento eran pacientes manejados en la unidad de radioterapia.

\section{Sobrevida del carcinoma espinocelular de la cavidad oral}

El tiempo medio de sobrevida fue 6,9 años y se demostró que el $75 \%$ de la muestra se mantuvo vivo al año, similar a estudio de Vallecillo y cols $^{2}$, con promedio de 5,7 años y $75 \%$ de pacientes vivos a los 2,5 años.

A los cinco años la sobrevida del presente estudio fue $46 \%$, cifra menor a lo entregado en Surveillance Epidemiology and End Result (SEER), $61 \%{ }^{18}$. Esta diferencia se puede asociar a mayor accesibilidad a terapia y posible detección más temprana. Además podrían afectar diferencias genéticas y medio ambientales exclusivas de cada población.

En Chile, existen pocos estudios en sobrevida de CEC. Capdeville y $\operatorname{cols}^{28}$, mostró mayor sobrevida que el presente estudio, $56,9 \%$ a los cinco años y $46,3 \%$ a los 10 años. La muestra de Capdeville incluyó otros tumores malignos, tales como de origen salival, los que generalmente tienen mayor sobrevida.

Arriagada y cols ${ }^{19}$, en Chile, reveló sobrevida de $58,4 \%$ a los cinco años en una muestra de 36 pacientes.

Otros autores reportan sobrevidas considerablemente más bajas. Campos y cols ${ }^{25}$, mostró $39 \%$ a los cinco años y Kruaysawat y $\operatorname{cols}^{26}, 18,2 \%$ en 519 pacientes.

$\mathrm{Al}$ relacionar las curvas de sobrevida entre géneros, los hombres con CEC tendieron a tener sobrevida más corta $(38,3 \%)$ que las mujeres $(56,6 \%)$. Esto concuerda con estudio de español ${ }^{2}$ en muestra similar.

Las curvas de sobrevida obtenidas en Chile, reflejan que la prevención del carcinoma espinocelular necesita mayor atención.

\section{Sobrevida y edad}

Al analizar las tres curvas de sobrevida según distintos grupos etarios, se observó diferencias significativas, al igual que lo analizado por Arriagada y cols ${ }^{19}$. La mejor sobrevida se obtuvo en pacientes menores de 45 años, seguido por grupo de 45-60 años y con sobrevida más baja en los mayores de 60 años. En el reporte SEER ${ }^{18}$, se encontró el mismo orden pero con porcentajes de sobrevida algo mayor.

Otros estudios ${ }^{4,33}$, mencionan que pacientes jóvenes pueden presentar menor sobrevida debido a que presentarían lesiones más agresivas.

\section{Sobrevida y localización}

Existe variada información con respecto a localización y sobrevida. Vallecillo y cols ${ }^{2}$, encontró que lesiones en comisura labial tendían a menor sobrevi$\mathrm{da}$, pero se atribuye al pequeño tamaño de la muestra $(2,7 \%$ de los casos). El mismo estudio demostró promedio de sobrevida de 2,5 años en lesiones del trígono retromolar, ubicación comparable en nuestro estudio con encía y reborde alveolar (promedio sobrevida 6 años).

La sobrevida más baja que arrojó este estudio, respecto a todas las ubicaciones fue piso de boca, concordante a lo entregado en SEER ${ }^{18}$, sin considerar faringe como localización. En estudio realizado en Brasil ${ }^{34}$, se encontró que el sitio con peor sobrevida era lengua.

\section{Sobrevida y tabaco}

A pesar que los pacientes no fumadores tuvieron mejor sobrevida que los fumadores, no hubo diferencia significativa entre las curvas, probablemente por el desequilibrio entre las muestras; mientras que $68,5 \%$ de la muestra fumaba, sólo $35,5 \%$ no lo hacía.

Abdul y $\operatorname{cols}^{35}$ y Campos y cols ${ }^{25}$, realizaron estudios que también demuestran una peor sobrevida en pacientes fumadores.

\section{Sobrevida y alcohol}

A pesar que el alcohol se considera factor de riesgo para el desarrollo del CEC, no está claro cómo afectaría la sobrevida. El presente estudio demostró menor sobrevida en pacientes bebedores pero no se encontraron estudios comparables. Se considera que el impacto de consumo de alcohol por sí solo, no afecta significativamente la sobrevida, es por esto que resulta más interesante analizarlo asociado al tabaco.

\section{Sobrevida y tratamiento}

Las curvas de Kaplan-Meier revelan que pacientes tratados con cirugía y radioterapia presentaron mejor sobrevida a los cinco años $(47,8 \%)$ que los que recibieron otro tipo de tratamiento; sobrevida de $40,3 \%$ a los cinco años.

Abdul y $\operatorname{cols}^{35}$, encontró que pacientes tratados 
con cirugía presentaron $38,5 \%$ de sobrevida a los cinco años, seguida por la cirugía con radioterapia, con $30,6 \%$.

Otro estudio ${ }^{25}$, concluyó que al realizar radioterapia postoperatoria la sobrevida disminuía.

Para lograr el diagnóstico en etapa precoz es de suma importancia realizar periódicamente un prolijo examen de cavidad bucal durante el examen clínico, especialmente en pacientes de alto riesgo; mayores de 60 años, fumadores, bebedores de alcohol y aquellos con antecedentes de CEC o con alguna otra neoplasia maligna.

\section{Agradecimientos}

A la Fundación Arturo López Pérez por estar siempre dispuestos a ayudarnos y entregar información necesaria para realizar este estudio. En especial a Clarita San Martín por su colaboración y ayuda en el departamento de radioterapia.

\section{Referencias}

1. García-García V, Bascones Martínez A. Cáncer oral: puesta al día. Avances en Odontoestomatología 2009;25:239-48.

2. Vallecillo M, Romero MN, Olmedo MV, Reyes C, Bustos V. Factors related to survival from oral cancer in an Andalusian population sample (Spain). Med Oral Patol Oral Cir Bucal. 2007;12:E518-23.

3. Riera P, Martínez B. Morbilidad y mortalidad por cáncer oral y faríngeo en Chile. Rev Med Chile 2005;133:55563.

4. Hirota S, Migliari D, Sugaya N. Oral squamous cell carcinoma in a young patient. Case report and literature review. An Bras Dermatol. 2006;81:251-4.

5. Millán A, González M. Tumores malignos de la mucosa oral. En Bagán (Ed.) Medicina Oral. Barcelona-España: Editorial Masson, 1995;187-99.

6. Khalili J. Oral cancer: risk factors, prevention and diagnostic. Exp Oncol. 2008;30:259-64.

7. Mehrotra R, Yadav S. Oral squamous cell carcinoma: Etiology, pathogenesis and prognostic value of genomic alterations. Indian J Cancer 2006;2:60-6.

8. Rodu B, Jansson C. Smokeless Tobacco and Oral Cancer: A Review of the Risks and Determinants. Crit Rev Oral Biol Med. 2004;15:252-63.

9. Silverman S. Demographics and occurrence of oral and pharyngeal cancers The outcomes, the trends, the challenge. JADA 2001;172:7s-11s.

10. Honorato J, Camisasca D, Esmeraldo da Silva L, Dias F, Silvestre de Faria P, Chaves S. Análise de sobrevida global em pacientes diagnosticados com carcinoma de células escamosas de boca no INCA no ano de 1999. Rev Bras Epidemiol. 2009;12:69-81.
11. Meza G, Muñoz JJ, Páez C, Cruz B, Aldape B. Carcinoma de células escamosas de cavidad bucal en un centro de tercer nivel de atención social en la ciudad de México. Experiencia de cinco años. Av. Odontoestomatol. 2009;25:19-28.

12. Cabral LA, Carvalho LF, Salgado JA, Brandão AA, Alameida JD. Gingival Squamous Cell: a Case Report. J Oral Maxillofac Res. 2010;1:1-6.

13. Hirota S, Braga F, Penha S, Sugaya N, Migliari D. Risk factors for oral squamous cell carcinoma in young and older Brazilian patients: A comparative analysis. Med Oral Patol Oral Cir Bucal. 2008;13:E227-31.

14. Rocha A. Cáncer oral: el papel del odontólogo en la detección temprana y control. Rev Fac Odontol Univ Antioq. 2009;21:112-21.

15. Durazzo MD, Araújo CEN de, Brandao Neto J de S, Potenza A de S, Costa P, Takeda F, et al. Clinical and epidemiological features of oral cancer in a medical school teaching hospital from 1994 to 2002: increasing incidence in women, predominance of advanced local disease, and low incidence of neck metastases. Clinics 2005;60:293-8.

16. Brener S, Jeunon FA, Barbosa A, Grandinetti H. Carcinoma de células escamosas bucal: uma revisão de literatura entre o perfil do paciente, estadiamento clínico e tratamento proposto. Revista Brasileira de Cancerologia 2007;53:63-9.

17. Sapp JP, Eversole LR, Wysocki G. Patología Oral y Máxilofacial Contemporáneas. Ed.1. Editorial Harcourt Brace. 1998. Capítulo 6 p:172-82.

18. Surveillance Epidemiology and End Result (SEER) [página de Internet] Cancer Statistics Review 1975-2008 [Visitado el 8 de agosto de 2011] Disponible en: http:// seer.cancer.gov/csr/1975_2008/sections.html

19. Arriagada O, Venegas R, Cantín M, Zavando D, Manterola C, Suazo I. Rol de las características clínicas e histológicas como factores pronósticos para la sobrevida en pacientes con carcinoma de células escamosas de la cavidad oral. Rev Chil Cir. 2010;62:441-8.

20. Salinas R, Marques S, Aparecido R, Rapoport A, Porto $\mathrm{O}$, de Andrade J. Epidemiologic Study of Squamous Cell Carcinoma of the Mouth and Oropharynx. Intl. Arch. Otorhinolaryngol. 2007;11:271-7.

21. Iamaroon A, Pattanaporn K, Pongsiriwet S, Wanachantararak S, Prapayasatok S, Jittidecharaks S, et al. Analysis of 587 cases of oral squamous cell carcinoma in northern Thailand with a focus on young people. Int J Oral Maxillofac Surg. 2004;33:84-8.

22. Álvarez E, Barbosa M, Gaviria AM. Comportamiento clínico-epidemiológico del carcinoma escamocelular bucal de pacientes tratados en el Hospital Universitario San Vicente De Paúl (HUSVP), Medellín, entre enero de 1990 y diciembre de 1996. Rev. Facultad de Odontología Universidad de Antioquia. 2005:16:26-32.

23. Guzmán P, Villaseca M, Antonio L, Araya J, Aravena $\mathrm{P}$, Cravero C, y cols. Carcinoma epidermoide oral y 
orofaringeo. Estudio clínico-patológico. Rev Chil Cir. 2011;63:250-6.

24. Al-Rawi N, Talabani N. Squamous cell carcinoma of the oral cavity; a case series analysis of clinical presentations and histological grading of 1,425 cases from Irak. Clin Oral Invest. 2008;12:15-8.

25. Montoro JR, Ricz HA, Souza L, Livingstone D, Melo $\mathrm{HD}$, Tiveron RC, et al. Prognostic factors in squamous cell carcinoma of the oral cavity. Rev Bras Otorrinolaringol. 2008;74:861-6.

26. Kruaysawat W, Aekplakorn W, Chapman R. Survival Time and Prognostic Factors of Oral Cancer in Ubon Ratchathani Cancer Center. J Med Assoc Thai 2010;93:278-84.

27. Sánchez J, Chamorro C, Esquivias J, Valencia E. Factores histopatológicos en el carcinoma lingual de células escamosas. Rev Esp Patol. 2006;39:99-104.

28. Capdeville F. Tumores malignos de la cavidad oral. Operación comando. Reconstrucción mandibular. Rev Chil Cir. 2005;57:7-18.

29. Ildstad S, Tollerud D, Bigelow ME, Remensnyder J. A Multivariate. Analysis of Determinants of Survival for Patients with Squamous Cell Carcinoma of the Head and Neck. Ann Surg. 1989;209:237-41.
30. Morelatto R, López S. Mortalidad por cáncer bucal en la provincial de Córdoba, República Argentina (período 1975-2000). Estudio comparativo con otras poblaciones. Med Oral Patol Oral Cir Bucal. 2006;1:E230-5.

31. Peña A, Arredondo M, Vila L. Comportamiento clínico y epidemiológico del cáncer de cavidad oral. Revista Cubana de Estomatología 2006;43:224-9.

32. Bórquez M, Capdeville F, Madrid A, Veloso M, Cárcamo M. Sobrevida global y por estadíos de 137 pacientes con cáncer intraoral. Experiencia del Instituto Nacional del Cáncer. Rev Chil Cir. 2011;63:351-5.

33. Mallet Y, Avalos N, Le Ridant AM, Gangloff P, Moriniere S, Rame JP, et al. Head and neck cancer in young people: a series of 52 SCCs of the oral tongue in patients aged 35 years or less. Acta Otolaryngol. 2009;129:1503-8.

34. Oliveira LR, Ribeiro-Silva A, Zucoloto S. Perfil de incidência e da sobrevida de pacientes com carcinoma epidermóide oral em uma populaçao brasileira. J Bras Patol Med Lab. 2006;42:385-92.

35. Abdul A, Saddki N, Naing NN, Abdullah N. Oral Cancer Survival among Malay Patients in Hospital Universiti Sains Malaysia, Kelantan. Asian Pacific J Cancer Prev. 2010;10:187-91. 